\title{
Pyrrhussieg bei der Finanzierung der Gesetzlichen Krankenversicherung
}

\section{WOLFRAM F. RICHTER}

Prof. Dr. Wolfram F. Richter ist Inhaber des Lehrstuhls VWL (Öffentliche Finanzen) an der TU Dortmund und Mitglied im Wissenschaftlichen Beirat beim Bundesministerium der Finanzen

Laut Koalitionsvertrag sollen die gesetzlichen Krankenkassen den kassenindividuellen Zusatzbeitrag zukünftig als prozentualen Satz vom beitragspflichtigen Einkommen erheben. Der Verhandlungsführer der SPD und Verfechter der Bürgerversicherung, Karl Lauterbach, feierte dieses Verhandlungsergebnis als das historische Ende der Kopfpauschale. Dieser Beitrag argumentiert, dass mit dem Ende der gesetzlichen Kopfpauschale auch die Bürgerversicherung in weite Ferne rückt. Die einkommensabhängige Bemessung der Zusatzbeiträge wird eine wettbewerbsgetriebene Spezialisierung von GKV und PKV begünstigen. Längerfristig wird die GKV nur noch das medizinisch Notwendige abdecken. Freiwillige Leistungen muss man dagegen privat versichern. Statt einer Annäherung von GKV und PKV wird man eine Auseinanderentwicklung erleben.

Der Verhandlungsführer der SPD und Verfechter der Bürgerversicherung, Karl Lauterbach, feierte die Verständigung mit den Unionsparteien über die zukünftige Finanzierung der Gesetzlichen Krankenversicherung (GKV) als das historische Ende der Kopfpauschale. Die Freude dürfte allerdings nicht von langer Dauer sein. Sie dürfte nur so lange währen, bis die Auswirkungen der Verständigung auf die GKV durchdacht wurden. Dann wird man begreifen, dass mit dem Ende der gesetzlichen Kopfpauschale auch die Bürgerversicherung in weite Ferne rückt.

Um diesen Pyrrhussieg zu verstehen, muss man zunächst den Begriff der Bürgerversicherung präzisieren und dann auch noch klären, mit welchen Reaktionen der gesetzlich Versicherten auf die zukünftige Verbeitragung in der GKV zu rechnen sein wird.

Der Begriff der Bürgerversicherung vereint zwei Gestaltungsprinzipien. Das eine Prinzip ist abgabenrechtlicher Art. Es postuliert eine Beitragserhebung nach der Leistungsfähigkeit, wie sie für die Erhebung von Steuern prägend ist. Es bedeutet, dass der Beitrag einkommensbezogen erhoben wird und nicht einkommensunabhängig wie bei einer Finanzierung mittels Kopfpauschale. Ferner bedeutet es, dass nicht nur Lohneinkommen unterhalb einer Beitragsbemessungsgrenze beitragspflichtig ist, sondern auch Lohneinkommen oberhalb der Bemessungsgrenze sowie sonstiges Einkommen aus Kapital, Vermietung usw. Das andere Gestaltungsprinzip drückt sich in der 
Namensgebung aus. Die Bürgerversicherung soll alle Bürger eines Landes gleich behandeln. Es soll insbesondere bei der Versicherung keinen Unterschied machen, ob eine Person abhängig beschäftigt ist, Beamtin oder Selbständige. Die Bürgerversicherung verkörpert also das Leitbild einer Versicherung, die sich durch leistungsgerechte Beitragserhebung und bürgerliche Gleichbehandlung auszeichnet.

Der Koalitionsvertrag sieht nun vor, dass die gesetzlichen Krankenkassen den kassenindividuellen Zusatzbeitrag zukünftig als prozentualen Satz vom beitragspflichtigen Einkommen erheben. Das ist der Teil der Verständigung, mit dem der Verhandlungsführer der SPD das Ende der Kopfpauschale begründet. Die Vereinbarung lässt sich in der Tat als einen kleinen Schritt in Richtung einer Bürgerversicherung deuten. Nicht nur der allgemeine Beitrag, sondern auch kassenindividuelle Zusatzbeiträge sollen zukünftig einkommensbezogen erhoben werden. ${ }^{1}$ Das steht im Einklang mit der Forderung nach leistungsgerechter Verbeitragung. Allerdings bleibt die Beitragsbemessungsgrundlage unverändert. Lohneinkommen oberhalb der Bemessungsgrenze sowie sonstiges Einkommen aus Kapital oder Vermietung wird weiterhin abgabenfrei sein. Vor allem aber wird der Kreis der gesetzlich Versicherten nicht ausgeweitet. Beamte, Selbständige und gut verdienende Arbeitnehmer werden sich weiterhin privat versichern können.

Gesundheitsrisiken werden also auch zukünftig außerhalb der GKV versichert, und diese Option wird sich ohne Verfassungsänderung nicht ausschließen lassen. Daher muss man darüber nachdenken, wie sich eine gesetzliche Umsetzung der Koalitionsvereinbarung auf das Verhältnis von gesetzlicher zu privater Krankenversicherung auswirkt. Wie im Weiteren eingehend begründet werden soll, dürfte die PKV der eigentliche Gewinner der Umsetzung sein. Die GKV wird im Wettbewerb der beiden Systeme längerfristig der Verlierer sein. Genauer wird der Abschluss einer privaten Zusatzversicherung das Schlupfloch sein, das die Menschen nutzen werden, um die Koalitionsvereinbarung zu unterlaufen. Konkret muss man mit folgenden Ausweichreaktionen rechnen, sollte die Koalitionsvereinbarung Gesetzeskraft erlangen.
Es werden vorzugsweise junge und überdurchschnittlich gut verdienende Mitglieder der GKV sein, die die umverteilende Wirkung einkommensabhängiger Beitragssätze nicht einfach hinnehmen. Sie werden lernen, sich der Umverteilung da zu entziehen, wo es gesetzlich möglich ist. Das ist der Bereich der freiwilligen Kassenleistungen. Die Kräfte des Wettbewerbs werden die gesetzlichen Kassen dazu bringen, Wahltarife anzubieten, die freiwillige Leistungen ausschließen, jedenfalls soweit dies praktikabel erscheint. Sofern die genannten Mitglieder solche Leistungen wert schätzen, werden sie sie auf privater Basis nachfragen. Die privaten Versicherungen werden sie zu günstigen Pauschalprämien anbieten, und zwar ohne die Berücksichtigung von Altersrückstellungen. Altersrückstellungen sind nur dann gefragt, wenn man sich gegen das altersbedingte Prämienanpassungsrisiko versichern möchte. Die Notwendigkeit

die Beitragssätze für die verbleibenden Mitglieder entsprechend steigen müssen. Das wird weitere Mitglieder veranlassen, dem Beispiel zu folgen. Das Ergebnis des Prozesses wird sein, dass nur solche GKV-Tarife den Wettbewerb überleben, die sich dadurch auszeichnen, dass sie auf die Versicherung freiwilliger Leistungen möglichst verzichten. Freiwillige Leistungen werden sich am Ende nur noch privat versichern lassen, und zwar zu einkommensunabhängigen Prämien, die das individuelle Gesundheitsrisiko widerspiegeln.

Das Ergebnis wird wenig den Vorstellungen entsprechen, an denen sich die Koalitionsverhandlungen orientiert haben. Die Bevölkerung wird längerfristig noch stärker als heute das medizinisch Notwendige mit der GKV in Verbindung bringen und freiwillige Leistungen mit der PKV. Die wettbewerbsgetriebene Spezialisierung von GKV und PKV wird nicht nur für die Wahrnehmung der Menschen prägend sein. Sie wird auch den Übergang zu einer gleichbehandelnden Bürgerversicherung endgültig verbauen. Man wird Beamten, Selbständigen und gutverdienenden Arbeitnehmern politisch immer weniger vermitteln können, warum sie ihre Privatversicherung aufgeben sollen, um in einer weniger wertgeschätzten gesetzlichen Versicherung Mitglied zu werden. Statt Gleichbehandlung durch behutsame Annäherung der Versicherungssysteme wird es die Zementierung bürgerlicher Ungleichbehandlung durch eine politisch falsche Weichenstellung geben.

Dieser Prognose wird nicht jeder Leser einfach zustimmen wollen. Er wird nach Einwänden suchen. So dürfte er erstens einwenden, dass es einkommensbezogene Beiträge auch schon vor Einführung des Gesundheitsfonds gegeben hat, ohne dass die skizzierten Ausweichhandlungen zum Problem wurden. Zweitens wird er darauf hinweisen, dass der Koalitionsvertrag eine Bestimmung enthält, die den Gesetzgeber explizit zum Ausgleich etwaiger Wettbewerbsverzerrungen verpflichtet. Drittens wird er die Bedeutung freiwilliger Leistungen für die GKV klein reden. Keiner der drei Einwände ist indessen geeignet, die Prognose substanziell zu widerlegen.

1 Einzuräumen ist, dass die einkommensbezogene Bemessung von Zusatzbeiträgen in der Wissenschaft unterschiedlich beurteilt wird. Sie wurde von Klaus Jacobs und Jürgen Wasem sogar empfohlen. Vgl. dieselben, 2013, Vier Jahre Gesundheitsfonds - ein Modell mit Zukunft?, Gesundheit und Gesellschaft Wissenschaft 13, S. 15-22. 
Richtig ist, dass es einkommensbezogene Beiträge auch schon vor Einführung des Gesundheitsfonds gab und dass es trotz größerer Beitragssatzunterschiede zu keiner nennenswerten Flucht der Mitglieder in billige Kassen kam. Die Wechselbereitschaft stieg erst in dem Augenblick, als nach Einführung des Gesundheitsfonds einkommensunabhän-

\section{Der Arbeitgeber wird weiterhin Zusatzbeiträge nicht mitfinanzieren: Er wird lediglich das Inkasso übernehmen.}

gige Zusatzbeiträge direkt beim Versicherten einzufordern waren. Die gesetzlichen Krankenkassen verbinden Wettbewerbsdruck nach dieser Erfahrung auch in erster Linie mit Pauschalprämien, die beim Versicherten eingefordert werden. Entsprechend kommentierten ihre Repräsentanten die Koalitionsvereinbarung mit großer Erleichterung. Sie verwiesen darauf, dass die einkommensbezogene Bemessung der Zusatzbeiträge ein Lohnabzugsverfahren beim Arbeitgeber notwendig macht. Damit entfalle das scharfe Preissignal der direkt beim Versicherten eingezogenen Zusatzbeiträge. ${ }^{2}$

Bei dieser Beurteilung wird indessen übersehen, dass die Zusatzbeiträge anders als früher allein vom Arbeitnehmer aufzubringen sind. Vor Einführung des Gesundheitsfonds kamen Arbeitgeber und Arbeitnehmer zu gleichen Teilen für den GKV-Beitrag auf. Daher halbierte sich auch der finanzielle Vorteil eines Kassenwechsels. ${ }^{3}$ Das ist nicht länger der Fall. Der Arbeitgeber wird, wie vom Koalitionsvertrag bestätigt, Zusatzbeiträge nicht mitfinanzieren. Er wird lediglich das Inkasso übernehmen. Für die Versicherten erhöht daher jeder Euro, der sich bei einem Kassenwechsel einsparen lässt, das eigene Einkommen. Vielleicht wird die Wechselbereitschaft nicht ganz so hoch sein wie bei pauschalen Zusatzbeiträgen, die direkt beim Versicherten erhoben werden. Der Grund könnte sein, dass Beitragssatzunterschiede ein schwächeres Preissignal darstellen als feste Eurobeträge. In jedem Fall wird aber die Wechselbereitschaft erheblich höher sein als vor Einführung des Gesundheitsfonds.

Auch der Hinweis, dass der Koalitionsvertrag eine Bestimmung enthält, die den Gesetzgeber zum Ausgleich einkommens- bedingter Wettbewerbsverzerrungen verpflichtet, kann nicht wirklich überzeugen. Die prognostizierten Ausweichhandlungen der gesetzlich Versicherten berühren ja den Wettbewerb zwischen GKV und PKV. Ein Ausgleich der Verzerrung würde einen systemübergreifenden Einkommensausgleich erfordern, für den jede verfassungsrechtliche Grundlage fehlt. Man könnte allenfalls einen Einkommensausgleich zwischen den gesetzlichen Krankenversicherungen erwägen. Der wird aber das Problem nicht beheben können. Vertretbar wäre er nur so weit, wie die Einkommensstruktur der Mitgliederbasis einen Wettbewerbsnachteil darstellt, auf den die Krankenkassen keinen Einfluss haben. Das war in den Jahren 2009 bis 2010 der Fall. Hintergrund war die so genannte Überforderungsklausel, die die Krankenkassen in der Anfangszeit des Gesundheitsfonds dazu zwang, grundsätzlich pauschal zu erhebende Zusatzbeiträge auf ein Prozent des beitragspflichtigen Einkommens zu begrenzen. Zu erwarten war, dass diese Überforderungsklausel bei Krankenkassen mit einkommensschwacher Mitgliederbasis eher greifen würde als bei der Konkurrenz. Man rechnete mit erhöhter Abwanderung der Versicherten, und zwar ohne dass die betroffenen Krankenkassen verantwortlich zu machen gewesen wären. ${ }^{4}$ Unter solchen Bedingungen würde ein Einkommensstrukturausgleich die wünschenswerte Wettbewerbsgleichheit wieder herstellen.

Die Situation ist anders zu beurteilen, wenn die Höhe der Zusatzbeitragssätze unmittelbar auf kassenspezifische Tarifpolitik zurückzuführen ist. Wenn ein Wahltarif den Katalog der freiwilligen Leistungen breit und großzügig abdeckt, sind hohe Zusatzbeitragssätze vorprogrammiert. Wenn sich dann junge und gut verdienende Mitglieder wegen der beschriebenen Umverteilungswirkung aus solchen Komforttarifen verabschieden, kann es nicht richtig sein, den Schwund im Abgabensubstrat systemisch auszugleichen. Man würde völlig unsinnige Anreize zur Ausweitung freiwilliger Leistungen setzen.

$\mathrm{Zu}$ guter Letzt ist der Versuch abzuwehren, die Bedeutung freiwilliger Leistungen für die GKV klein zu reden. Hinweise in der Literatur, dass 95 Prozent der GKV-Leistungen Pflichtleistungen darstellen, sind irreführend. ${ }^{5}$ Sie igno- rieren die Spielräume, die es beim Versorgungsmanagement gibt. Versorgungsmanagement basiert auf der Grundidee, Arzt- und Therapiewahl auf freiwilliger Basis einzuschränken. Es findet seinen Ausdruck in Hausarzt- und Select-Tarifen. Letzteres sind Tarife, bei denen sich der Versicherte verpflichtet, im Krankheitsfall vertraglich gebundene Leistungserbringer aufzusuchen. International gilt Versorgungsmanagement als der Schlüssel zu einem Gesundheitswesen, das eine medizinisch hochwertige Versorgung kostengünstig ermöglicht. Nach Untersuchungen aus der Schweiz lassen sich auf diesem Weg bei gleichem medizinischem Nutzen Kosteneinsparungen von 20 bis 37 Prozent erzielen. ${ }^{6}$ Selbst wenn nur 20 Prozent realisierbar sind, lässt sich ein durchschnittlicher Zusatzbeitrag von bis zu 3,65 Prozent des beitragspflichtigen Einkommens durch Versorgungsmanagement vollständig vermeiden. Denn 3,65 Prozent sind 20 Prozent der Summe von 14,6 und 3,65 Prozent. Hierbei stehen die 14,6 Prozent für den allgemeinen paritätisch finanzierten Beitragssatz.

In der GKV lassen sich also medizinisch nicht notwendige und damit als freiwillig verursacht zu begreifende Kosten von erheblichem Umfang einsparen. Der Leser mag sich fragen, warum das nicht schon längst geschehen ist und warum Versorgungsmanagement in wenigen Ländern so unterentwickelt ist wie in Deutsch-

2 Die Hoffnung auf reduzierten Wettbewerbsdruck wird indessen mit Sorge um das Wohl der Patienten bemäntelt. So soll der DAK-Chef Herbert Rebscher gesagt haben, dass der „bisherige Anreiz, Zusatzbeiträge um jeden Preis zu vermeiden, vor allem zu Lasten der Patienten ging und die Gefahr der Risikoselektion barg". Zitiert nach Anno Fricke, Ärzte Zeitung online, 22. Nov. 2013, Abfrage am 29. Nov. 2013: http:// www.aerztezeitung.de/politik_gesellschaft/ gp_specials/bundestagswahl-2013/ article/850562/vorwaerts-vergangenheit-ag-gesundheit-kippt-kopfpauschale. html?sh=1\&h=296814126)

3 Wissenschaftlicher Beirat beim Bundesministerium der Finanzen, 2004, Nachhaltige Finanzierung der Renten- und Krankenversicherung, Band 77, Schriftenreihe des Bundesministeriums der Finanzen.

4 Sachverständigenrat zur Begutachtung der wirtschaftlichen Entwicklung, 2006, Widerstreitende Interessen - Ungenutzte Chancen, Jahresgutachten 2006/07.

5 So z.B. F. Buchner, und J. Wasem, 2003, Needs for Further Improvement: Risk Adjustment in the German Health Insurance System, Health Policy 65, 21-35.

6 Leu, R. E., F. Rutten, W. Brouwer, C. Rütschi and P. Matter, 2008, The Swiss and the Dutch Health Care Systems Compared: A Tale of Two Systems, Nomos Verlag, BadenBaden, S. 114 
land. Der Ökonom wird verschiedene Regulierungsdefizite im Wettbewerb der Krankenkassen verantwortlich machen. An erster Stelle wird er das stark eingeschränkte Recht der Krankenkassen nennen, mit medizinischen Leistungserbringern selektiv zu kontrahieren. An einer vorausplanenden Bedarfssteuerung bei den von Krankenhäusern erbrachten Leistungsmengen fehlt es in Deutschland überhaupt, wie noch jüngst die OECD monierte. ${ }^{7}$ Es fehlte aber auch bis jüngst an Anreizen für die gesetzlich Versicherten, auf freiwillige Leistungen tariflich zu verzichten. Wie schon erwähnt, teilten sich vor Einführung des Gesundheitsfonds die Versicherten sämtliche Beitragsersparnisse mit dem Arbeitgeber. Unter solchen Bedingungen sind für den Arbeitnehmer verständlicherweise diejenigen Versicherungstarife am attraktivsten, die den großzügigsten Leistungskatalog versprechen. Erst mit der Einführung des Gesundheitsfonds wurde die systematische Subventionierung freiwilliger Leistungen durch den Arbeitgeber gestoppt. Gleichwohl haben sich die Marktkräfte noch nicht im wünschenswerten Maße entfalten können. Die wichtigste Ursache muss in dem viel zu hoch fixierten allgemeinen Beitragssatz von 14,6 plus 0,9 Prozent gesehen werden. Er hat dazu geführt, dass die Mittel, die über den Gesundheitsfonds verteilt werden, zu reichlich fließen. Den gesetzlichen Krankenkassen fehlt überwiegend die Notwendigkeit, Zusatzbeiträge zu erheben. Damit fehlt den Versicherten aber derzeit auch der Anreiz, Kosten sparende Wahltarife nachzufragen.

Die möglichen Einwände gegen die prognostizierte Spezialisierung von GKV und PKV lassen sich also entkräften. Es bleibt dabei, dass die Politik damit rechnen muss, dass sich die GKV längerfristig in Richtung einer Versicherung des medizinisch Notwendigen entwickelt, die von all denjenigen gemieden wird, die die gesetzliche Freiheit der Wahl besitzen und die mehr als nur das medizinisch Notwendige abgesichert sehen wollen. Statt einer Annäherung von GKV und PKV wird es eine Auseinanderentwicklung geben.

Das Problem ist, dass die Anhänger der Bürgerversicherung zu viel politisch erzwingen wollen. Sie wollen sowohl eine leistungsgerechte Beitragserhebung verwirklichen als auch eine versicherungsrechtliche Gleichbehandlung aller Bürger. Vor dem Hintergrund des in Deutschland gewachsenen dualen Sys- tems der Versicherung von Gesundheitsrisiken ist aber beides nicht zusammen zu haben. Die Politik täte gut daran, sich auf eine Priorisierung zu verständigen. Aus ökonomischer Sicht fällt eine solche Priorisierung leicht. Die bürgerliche Gleichbehandlung muss gegenüber einer Form der Beitragserhebung vorgezogen werden, die selbst noch bei freiwilligen Zusatzleistungen auf Einkommensbezogenheit insistiert. Der Grund ist nicht zuletzt in den Herausforderungen zu sehen, die der medizinisch-technische Fortschritt und der demografische Wandel für die Gesellschaft mit sich bringen.

Es ist davon auszugehen, dass der medizinisch-technische Fortschritt das Angebot an medizinisch nicht notwendigen Gesundheitsleistungen stetig wachsen lässt. Die Fantasie der Leistungserbringer

\section{Die Einigung der Koalitions- parteien auf einkommensab- hängige Zusatzbeiträge muss als historischer Fehler begriffen werden.}

die Beitragslast von Versicherten mit kleinem Einkommen relativ sinken lassen und deren Nachfrage nach Gesundheitsleistungen dadurch zusätzlich anheizen. Allein die einkommensunabhängige Bemessung von Zusatzbeiträgen kann die Übernachfrage stoppen. Vor diesem Hintergrund muss die Einigung der Koalitionsparteien auf einkommensabhängige Zusatzbeiträge als historischer Fehler begriffen werden.

Eine überzeugende Reformstrategie sähe wie folgt aus. In einem ersten Schritt würde man sich darauf verständigen, dass eine Gleichbehandlung aller Bürger bei der Versicherung von Krankheitskosten als das prioritäre Langfristziel der Gesundheitspolitik zu gelten hat. Die Anerkennung dieses Ziels würde bedeuten, dass zwischen den gesetzlich Versicherten einerseits und den Beamten, Selbständigen und nicht gesetzlich versicherten Arbeitnehmern andererseits ein Interessensausgleich gefunden werden muss. Da eine einkommensbezogene Beitragsbemessung für die GKV prägend ist so wie die einkommensunabhängige Prämienbemessung für die PKV, müsste die zukünftige Bürgerversicherung beide Tra-

wird diese Entwicklung befördern, und die Alterung der Bevölkerung wird die Nachfrage auf natürliche Weise steigen lassen. Eine solche Entwicklung kann man nicht einfach laufen lassen. Sie muss sich vielmehr in geordneten Bahnen vollziehen. Der Komplex der medizinisch nicht notwendigen Leistungen wird nur dann nicht zu einer Wohlstandsbürde, wenn es gelingt, die verursachten Kosten den Nutznießern anzulasten. Die ethischen Argumente, die bei medizinisch Notwendigem gegen eine verursachungsgerechte Anlastung der Kosten sprechen, sind jedenfalls auf freiwillige Leistungen nicht anwendbar.

Derzeit werden in der GKV die Kosten der medizinisch nicht notwendigen Leistungen nicht verursachungsrecht finanziert. Die Politik scheint das Problem nicht zu kümmern. Die Anhänger der Bürgerversicherung sorgt es erst recht nicht. Sie streben nach Finanzierungsgerechtigkeit und weniger nach effizienter Nutzung knapper volkswirtschaftlicher Ressourcen. Das Finanzierungsmodell der Bürgerversicherung würde die Fehlsteuerung im Gesundheitswesen noch verstärken. Die Verbreiterung der Bemessungsgrundlagen von Beiträgen und Zusatzbeiträgen würde ditionen in sich vereinen. Dafür wurden mit der Einführung des Gesundheitsfonds die Weichen in der GKV richtig gestellt. Lediglich der allgemeine Beitrag wird zurzeit einkommensbezogen erhoben, der kassenindividuelle Zusatzbeitrag dagegen grundsätzlich pauschal.

Statt diese Weichenstellung ohne Not zu blockieren, wäre der Ansatz der dualen Beitragsfinanzierung konsequent weiterzuentwickeln. Der nächste Schritt sollte in einer Verständigung auf die Regel liegen, dass in der GKV das medizinisch Notwendige einkommensabhängig und die freiwilligen Leistungen einkommensunabhängig zu verbeitragen sind. Welche Leistungen als medizinisch notwendig zu gelten haben, könnte dabei wie bisher von dem Gemeinsamen Bundesausschuss festgelegt werden.

Damit die gesetzlichen Krankenkassen die Rolle eines Sachwalters der Versicherteninteressen annehmen und innovativ entwickeln, sind drei Bedingungen zu erfüllen. Erstens ist politisch zu akzeptieren, dass Versorgungsmanagement nicht zu den Dingen zählt, die durch den Gesetz-

7 OECD (2013), Managing hospital volumes - Germany and experiences from OECD countries. 
geber oder den Gemeinsamen Bundesausschuss zu regeln sind. Zweitens müssen die Krankenkassen den vertragsrechtlichen Spielraum erhalten, um Wahltarife zu entwickeln, die den Versicherten unterschiedlichen Versorgungskomfort bieten. Das bedeutet konkret, dass es den Krankenkassen gestattet sein muss, mit den Erbringern medizinischer Leistungen selektiv zu kontrahieren. Drittens müssen die Krankenkassen durch wettbewerblichen Druck dazu gebracht werden, im Bereich des Versorgungsmanagements innovative Tarifpolitik zu betreiben. Dafür sind pauschale Zusatzbeiträge notwendig. Nur sie geben den Versicherten unverfälschte Anreize, sich in der Tarifwahl für oder gegen Versorgungskomfort zu entscheiden. Solange es die herkömmliche Trennung von GKV und PKV gibt, sollten die Mittel im Gesundheitsfonds vom Gesetzgeber so gesteuert werden, dass sich alle gesetzlichen Krankenkassen gezwungen sehen, minimale, aber pauschale Zusatzbeiträge zu erheben.

In einem dritten und letzten Schritt ist die gleichbehandlungsorientierte Bürgerversicherung zu schaffen. Sie zeichnet sich dadurch aus, dass die gesetzlichen und privaten Versicherungen gleichberechtigt um Versicherte konkurrieren und dass die Bürger frei zwischen gesetzlichen und privaten Versicherern wählen dürfen. Es wird am Ende nicht länger gesetzliche und private Vollversicherungen geben, sondern nur noch eine gesetzlich verfügte Basisversicherung und daneben freiwillige Zusatzversicherungen. ${ }^{8}$ Jeder Bürger wird verpflichtet sein, für das medizinisch Notwendige die Basisversicherung abzuschließen. Nach der im zweiten Schritt entwickelten Regel liegt es nahe, Basisversicherungen über den Gesundheitsfonds zu finanzieren und damit einkommensbezogen. Der erläuterte Interessenausgleich zwischen den gesetzlich und privat Versicherten kann es indessen erforderlich machen, neben den Zusatzversicherungen einen Teil der Basisversicherung einkommensunabhängig zu verbeitragen. ${ }^{9}$ Das wäre allerdings dann politisch auszuhandeln. In diesem Zusammenhang könnte sich die Frage nach einem steuerfinanzierten Sozialausgleich stellen.

8 Vgl. Henke, K.-D. und W.F. Richter, 2009, Zur Zukunft des Gesundheitsfonds, Wirtschaftsdienst 89, 727 - 732 .

9 Vgl. Richter, W.F., 2009, Zur zukünftigen Finanzierung der Gesundheitsausgaben in Deutschland, Perspektiven der Wirtschaftspolitik 10, $469-479$.

\section{Der Patient im Internet}

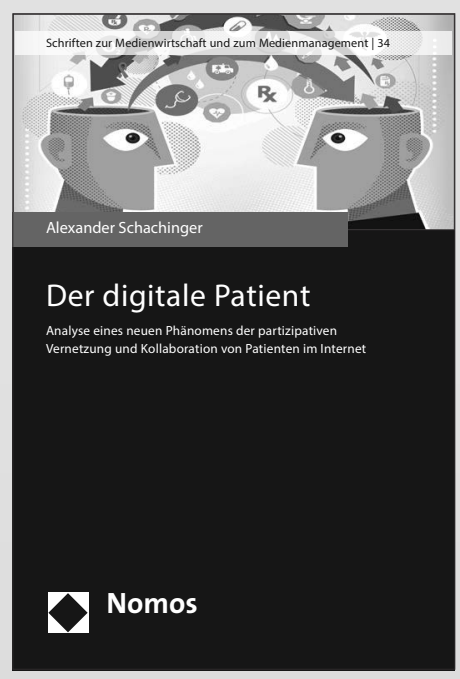

Der digitale Patient

Analyse eines neuen Phänomens der partizipativen Vernetzung und Kollaboration von Patienten im Internet

Von Alexander Schachinger

2014, 183 S., brosch., 34,- $€$

ISBN 978-3-8487-0961-8

(Schriften zur Medienwirtschaft und zum Medienmanagement, $B d$. 34)

www.nomos-shop.de/21822

Geschätzte 40 Millionen Personen im deutschsprachigen Raum nutzen bei Krankheitsfragen das Internet zur Recherche, zum Austausch und zu gemeinsamer Kollaboration. Wie schaut die Welt der medizinischen Symptome, Diagnosen, Therapien und gemeinsamer Erfahrungen von Patienten im Internet aus?

Die hier neu entstehenden digitalen Möglichkeiten für Patienten wachsen in ihrer Nutzung deutlich an, aber aktuell völlig unvernetzt mit dem traditionellen Gesundheitssystem. Was sagt der Forschungsstand zum Thema Patienten im Internet? Welche Foren, Apps und Anwendungen für Patienten entwickeln sich aktuell im Internet, und wie können diese, wenn überhaupt, mit den medizinischen Versorgungsstrukturen verknüpft werden?

Dieses Buch gibt eine erste Zusammenfassung im Bereich der Medizin im Internet und stellt sie dem deutschen Gesundheitssystem gegenüber. Knapp 2000 „E-Patienten“ wurden zu diesen Trends online befragt. Ihre Antworten sowie Chancen und Risiken des Phänomens „E-Patient“ werden als Fazit besprochen.

Bestellen Sie jetzt telefonisch unter 07221/2104-37. Portofreie Buch-Bestellungen unter www.nomos-shop.de

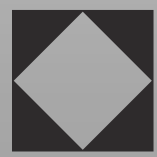
Nomos 